\title{
Bubble dynamics and size distributions during focused ultrasound insonation
}

\author{
Xinmai Yang ${ }^{\text {a) }}$ \\ National Center for Physical Acoustics, University of Mississippi, 1 Coliseum Drive, \\ University, Mississippi 38677 \\ Ronald A. Roy and R. Glynn Holt \\ Department of Aerospace and Mechanical Engineering, Boston University, Boston, Massachusetts 02215
}

(Received 24 April 2004; revised 4 October 2004; accepted 5 October 2004)

\begin{abstract}
The deposition of ultrasonic energy in tissue can cause tissue damage due to local heating. For pressures above a critical threshold, cavitation will occur, inducing a much larger thermal energy deposition in a local region. The present work develops a nonlinear bubble dynamics model to numerically investigate bubble oscillations and bubble-enhanced heating during focused ultrasound (HIFU) insonation. The model is applied to calculate two threshold-dependent phenomena occurring for nonlinearly oscillating bubbles: Shape instability and growth by rectified diffusion. These instabilities in turn are shown to place physical boundaries on the time-dependent bubble size distribution, and thus the thermal energy deposition. (C) 2004 Acoustical Society of America.
\end{abstract}

[DOI: $10.1121 / 1.1823251]$

PACS numbers: 43.35.Wa, 43.80.Gx, 43.80.Sh [AJS]

Pages: $3423-3431$

\section{INTRODUCTION}

Research on the effects of therapeutic ultrasound dates back at least 70 years. Biological, physical, and chemical effects due to high intensity ultrasound, sometimes coupled with cavitation activity, have been observed by several researchers who essentially established the field of therapeutic ultrasound. ${ }^{1-3}$ In addition to causing mechanical damage, cavitation has been observed to affect and, under the right conditions, enhance heat deposition from ultrasound. ${ }^{4-9}$

The preceding list of work on bubble-enhanced heating was reviewed in Holt and Roy, ${ }^{10}$ who also observed bubbleenhanced heating in an agar-based tissue-mimicking phantom from focused MHz-frequency ultrasound. Their findings were characterized by a threshold dependence and enhanced, but often erratic heating above the threshold. They also modeled the two dominant mechanisms for bubble-enhanced heating: Viscous dissipation from bubble oscillations and absorption of the sound emitted by oscillating bubbles. Quantitative estimates for thermal power deposition from a single oscillating bubble were on the order of $10 \mathrm{~mW}$, compared to $\sim 1 \mathrm{~W}$ from absorption of the incident ultrasound waves. Though their findings established quantitatively that heat deposition from cavitation could account for their enhanced heating measurements, neither their model nor their experimental results (nor other groups results) could provide any guidance as to the bubble sizes responsible for the enhanced heating. We will return to this point.

Edson ${ }^{11}$ extended the work of Holt and Roy by adding a passive cavitation detector (PCD) $)^{12}$ to monitor the presence of bubbles. His experimental results showed that the PCD detected (by detecting broadband acoustic emissions above 2 $\mathrm{MHz}$ with a primary field of $1 \mathrm{MHz}$ ) inertial cavitation co-

\footnotetext{
a) Author to whom correspondence should be addressed; electronic mail: xmyang@olemiss.edu
}

incident with enhanced heating. In addition, he calculated the bubble-related power deposition in a reasonable parameter space, and, by estimating the number of bubbles in the gel to be on the order of 10-100, he could account for the observed cavitation-enhanced temperature rise.

In recent work, Bailey et al. ${ }^{13}$ studied the effect of overpressure on focused ultrasound lesion shapes in vitro. They found at high intensity levels, lesions formed with no overpressure, were tadpole shaped, and shifted into the prefocal region, but lesions formed with high overpressure remained cigar shaped. They concluded this result supported the hypothesis that bubbles contributed to the tadpole-shaped lesion distribution.

An in vivo experimental investigation of bubbleenhanced heating is reported by Sokka et al. ${ }^{14}$ These authors monitored lesion formation in rabbit thigh while monitoring temperature via magnetic resonance imaging (MRI). They observed a faster temperature rise within the first few seconds and higher overall temperature in the bubble-enhanced exposures than the sonications without bubble formation, consistent with the observations in Ref. 10. The lesions created with the gas-bubble-enhanced heating exposures were two to three times larger by volume, consistently more spherical in shape and closer to the transducer than the control exposures, in accordance with the observations reported in Ref. 13.

Hilgenfeldt et al. ${ }^{15,16}$ modeled the energy deposited from bubbles of $\mu \mathrm{m}$ size and driven by diagnostic ultrasound pulses. They considered the effect of viscous dissipation and acoustic emissions from bubbles allowed to oscillate nonlinearly. They reported that the strong absorption of the acoustic radiation from nonlinearly oscillating bubbles results in a temperature rise as high as $100 \mathrm{~K}$ for driving pressure around $3 \mathrm{MPa}$ at a distance of $1 \mu \mathrm{m}$ from the bubble surface. Chavrier et al. ${ }^{17}$ also investigated the emissions from nonlinearly oscillating bubbles and the resulting absorption of the 
sound; a "global attenuation coefficient" is used in their energy equation to account for the presence of the bubbleenhanced heating owing to bubble acoustic emissions. They used their model to predict prefocal lesion growth when the bubble density exceeds 500 bubbles per cubic mm. Both of these numerical models employed an ideal gas assumption with a uniform temperature for the bubble interior dynamics. Neither of these models considered the effects of varying viscosity. Finally, neither of these models includes bubble shape instabilities or growth/dissolution via gaseous mass diffusion.

Though not the subject of the present paper, we note that our results here will be generally applicable to the occurrence of cavitation-related bioeffects other than enhanced heating. Thus, we note here that acoustically induced cavitation has been shown to play an important role in hemolysis, ${ }^{18-21}$ hemostasis, ${ }^{22-24}$ and drug transport, ${ }^{25}$ especially in the context of thrombolysis. ${ }^{26-31}$

Though the importance of cavitation bubbles in the context of therapeutic ultrasound is thus well established, any attempt to quantitatively account for the contributions of bubbles to the heating (or any other effect) must rely on an inference of the bubble size distribution, as bubble dynamics at a fixed driving frequency is very sensitive to the bubble equilibrium radius. Since such a distribution is difficult to measure, we turn our focus toward calculating the bubble size distribution by incorporating shape and growth instabilities into a model for bubbles in therapeutic ultrasound. Taken together, these two threshold-dependent instabilities will define an allowed bubble size distribution.

\section{BUBBLE DYNAMICS MODEL}

A comprehensive review of bubble dynamics may be found in The Acoustic Bubble. ${ }^{32}$ In what follows, a Newtonian fluid bubble dynamics model is assumed. We integrate the Newtonian model over a large viscosity range, from $0.001 \mathrm{~Pa}$ s to $0.5 \mathrm{~Pa}$ s. The choice of a large range of viscosity is to supplement this Newtonian fluid assumption; in reality we may expect tissue to behave as a viscoelastic material, and one of the features of such materials is shear thinning.

\section{A. Radial motion equation}

Throughout this work, the radial equation of motion we use is the so-called Keller-Miksis equation which was first derived by Keller and Miksis, ${ }^{33,34}$ and has the form,

$$
\begin{aligned}
(1- & \left.\frac{\dot{R}}{c_{L}}\right) R \ddot{R}+\frac{3}{2}\left(1-\frac{1}{3} \frac{\dot{R}}{c_{L}}\right) \dot{R}^{2} \\
= & \frac{1}{\rho}\left(1+\frac{\dot{R}}{c_{L}}+\frac{R}{c_{L}} \frac{d}{d t}\right) p_{B} \\
& -\frac{1}{\rho}\left(1+\frac{\dot{R}}{c_{L}}\right)\left(p_{\infty}-p_{s}\left(t+\frac{R}{c_{L}}\right)\right),
\end{aligned}
$$

where dots denote time derivatives, $R$ is the bubble radius, $c_{L}$ is the sound speed in the surrounding medium, $\rho$ is the density of surrounding medium, $p_{\infty}$ is the ambient pressure, $p_{s}(t)$ is the applied acoustic field pressure, and $p_{B}$ is the pressure on the surrounding medium side of the interface between the medium and the bubble. $p_{B}$ is related to the internal pressure $p$ of bubble by the following formula:

$$
p=p_{B}+\frac{2 \sigma}{R}+4 \mu \frac{\dot{R}}{R},
$$

where $\sigma$ is the surface tension coefficient, and $\mu$ is the shear dynamic viscosity. The sound field we chose in this study is sinusoidal:

$$
p_{s}(t)=p_{a} \cos \omega t,
$$

where $p_{a}$ is the acoustic amplitude and $\omega$ is the driving angular frequency. Most therapeutic applications of ultrasound rely on rather long tone bursts, approximating continuouswave conditions. Our model is not limited to sinusoidal forcing, but experimental and numerical results show that with our experimental material parameters, sound fields in tissue and tissue-mimicking media are very weakly nonlinear, therefore the harmonics of $\omega$ are unimportant for the bubble dynamics for the current applications.

\section{B. Internal pressure}

To solve the above radial equation, the internal pressure of bubble is needed. A model for the internal temperature and pressure of the bubble is described in detail in Prosperetti et al. ${ }^{35}$ and Kamath et al. ${ }^{36}$ This model accounts for heat transport by convection and conduction to and from the surrounding medium. The model was derived by assuming an ideal gas and spatial uniformity of the gas pressure inside the bubble. The internal pressure $p$ can be found by integrating:

$$
\dot{p}=\frac{3}{R}\left[\left.(\gamma-1) K \frac{\partial T}{\partial r}\right|_{R}-\gamma p \dot{R}\right],
$$

where $\gamma$ is the ratio of the specific heats of the gas and $K$ is the gas thermal conductivity. The gas temperature field $T(r, t)$ is obtained from

$$
\frac{\gamma}{\gamma-1} \frac{p}{T}\left(\frac{\partial T}{\partial t}+v \frac{\partial T}{\partial r}\right)=\dot{p}+\frac{1}{r^{2}} \frac{\partial}{\partial r}\left(\kappa r^{2} \frac{\partial T}{\partial r}\right)
$$

with

$$
v=\frac{1}{\gamma p}\left[(\gamma-1) K \frac{\partial T}{\partial r}-\frac{1}{3} r \dot{p}\right],
$$

where $v$ is the radial velocity of the gas inside of the bubble. The temperature boundary condition for a relatively cold liquid, such as water at normal ambient temperature, is,

$$
T[r=R(t), t]=T_{\infty},
$$

where $T_{\infty}$ is the undisturbed liquid temperature.

The solution of this set of equations of motion (1)-(7) requires numerical integration. Kamath and Prosperetti developed a Galerkin spectral method which we employ in this work. The ODE solver ODE15s in MATLAB is used to solve the resulting set of ODEs. 


\section{Stability of a spherical bubble}

An oscillating spherical bubble can also develop shape oscillations. An instability to wavy surface perturbations will cause shape oscillations of a spherical bubble. Plesset, ${ }^{37}$ Eller and Crum, ${ }^{38}$ Prosperetti, ${ }^{39}$ Hilgenfeldt et al.,${ }^{40}$ and Hao and Prosperetti ${ }^{41}$ have modeled the stability of a spherical surface using spherical harmonics to describe the perturbations. Consider a small axisymmetric distortion of the spherical interface $R(t)$,

$$
r=R(t)+a_{n}(t) P_{n}(\theta),
$$

where $P_{n}$ is the Legendre polynomial of degree $n$ and $a_{n}(t)$ is the amplitude. The shape distortion consists of a superposition of surface oscillations whose amplitudes are given by $a_{n}(t)$. The problem is to determine how the $a_{n}(t)$ behave.

Holt and Gaitan ${ }^{42}$ and Gaitan and Holt ${ }^{43}$ have reported experimental observations of the onset of shape instability for a bubble in water. A comparison of those experimental results with predictions (Hao and Prosperetti ${ }^{41}$ and Prosperetti and $\mathrm{Hao}^{44}$ ) shows quantitative agreement regarding mode $n$ observed and dependence on bubble size for all models of bubble oscillation. For the radial model Eq. (1), and with the following approximations (9)-(12), Hao and Prosperetti ${ }^{41}$ document that good quantitative agreement can be expected in the single bubble sonoluminescence range, corresponding to inertially collapsing bubbles where the driving frequency is around $20 \mathrm{kHz}$ and driving pressure is $0.8-1.4 \mathrm{~atm}$. is

The equation governing the time evolution of the $a_{n}(t)$

$$
\ddot{a}_{n}+B_{n}(t) \dot{a}_{n}-A_{n}(t) a_{n}=0
$$

with

$$
\begin{aligned}
A_{n}(t)= & (n-1) \frac{\ddot{R}}{R}-\frac{\beta_{n} \sigma}{\rho R^{3}} \\
& +\frac{2 \nu \dot{R}}{R^{3}}\left[-\beta_{n}+n(n+2)(n-1) \frac{1}{1+2 \delta / R}\right] \\
B_{n}(t)= & \frac{3 \dot{R}}{R}+\frac{2 \nu}{R^{2}}\left[-\beta_{n}+\frac{n(n+2)^{2}}{1+2 \delta / R}\right]
\end{aligned}
$$

where $\beta_{n}=(n-1)(n+1)(n+2), \nu$ is the kinematic viscosity, $a_{n}$ is the amplitude of the $n$th surface mode, and the boundary layer thickness $\delta=\min (\sqrt{\nu / \omega}, R / 2 n)$ (Prosperetti, ${ }^{45}$ Brenner et al. ${ }^{46}$ and Hilgenfeldt et al. ${ }^{40}$ ) The ordinary differential Eq. (9) can then be solved numerically using numerical values of $R, \dot{R}$, and $\ddot{R}$ from Eq. (1). To finally determine the threshold for breakup plotted in the figures, the criterion $\max \left(\left|a_{n}(t)\right| / R(t)\right) \geqslant 1$ is used for breakup via shape instability.

\section{Rectified diffusion}

The shape instability threshold is not enough to yield the bubble size. For a shape-stable bubble, its equilibrium radius may grow, shrink, or in certain rather rare cases remain stable because of rectified diffusion (Eller and Flynn). ${ }^{47}$ To determine the growth or dissolution of bubbles we integrate the following equation (Fyrillas and Szeri): ${ }^{48}$

$$
\begin{aligned}
\frac{d R_{0}}{d t}= & R_{0} \omega_{0} \frac{R_{g} T_{\infty} \rho}{M p_{\infty}} \frac{1}{P e}\left(1+\frac{4 \sigma}{3 R_{0} p_{\infty}}\right)^{-1} \\
& \times \frac{\left(c_{i}-c_{0}\left\langle p_{G}^{*}(\tau)\right\rangle_{\hat{\tau}}\right)}{\int_{0}^{\infty} \frac{d U}{\left\langle\left(3 U+x^{3}(\tau)\right)^{4 / 3}\right\rangle_{\hat{\tau}}}}
\end{aligned}
$$

where $R_{g}$ is the universal gas constant, $R_{0}$ is the equilibrium radius of bubble, $\omega_{0}$ is the natural frequency of the bubble, $T_{\infty}$ is the temperature of the surrounding liquid, $\rho$ is the density of liquid, $M$ is the molecule weight of gas inside the bubble, $p_{\infty}$ is the ambient pressure, $P_{e}$ is the Péclet number, $\sigma$ is the surface tension, $c_{i}$ is the dissolved gas concentration, $c_{0}$ is the saturation concentration in the liquid, $p_{G}^{*}$ is the dimensionless pressure inside the bubble, $\tau$ is the dimensionless time defined as $t=\tau / \omega_{0}, x$ is the dimensionless bubble radius defined as $R=x R_{0}$, and $U$ is the dimensionless radial coordinate in the liquid, and angled brackets denote a fourthorder time average. The validity of this method depends on the Péclet number being large, which can be easily satisfied in most cases. The rectified diffusion threshold is given when the growth rate is zero

$$
c_{i}-c_{0}\left\langle p_{G}^{*}(\tau)\right\rangle_{\hat{\tau}}=0,
$$

which is identical to Eller and Flynn's result. ${ }^{47}$

\section{RESULTS}

We chose the range of viscosity from $0.001 \mathrm{~Pa}$ s to 0.5 Pas for simulations, which covered most of the possible viscosity coefficients encountered in human soft tissue. We investigate a bubble size range from $0.01 \mu \mathrm{m}$ to $50 \mu \mathrm{m}$. The driving frequency used in all the simulations is $1 \mathrm{MHz}$. Other parameters used in the simulation are $c_{L}=1600 \mathrm{~m} / \mathrm{s}$, $\sigma=0.0725 \mathrm{~N} / \mathrm{m}, T_{\infty}=20^{\circ} \mathrm{C}, p_{\infty}=1 \mathrm{~atm}$, and $\rho=1100 \mathrm{~kg} / \mathrm{m}^{3}$. These values correspond to the properties of tissuemimicking phantoms used in our experiments. ${ }^{10,11,49,50}$ For more details on the calculations and other results, the reader is referred to Ref. 50.

\section{A. Stability boundaries}

Figures 1-3 show overviews of the bubble dynamics for acoustic pressures $p_{a}=1.0 \mathrm{MPa}$ and $2.5 \mathrm{MPa}$, at an excitation frequency of $1 \mathrm{MHz}$ (for more details and calculations the reader is referred to Yang).$^{50}$ The threshold for breakup via shape instability (labeled SI, calculated for the most unstable mode at each bubble size and viscosity), the threshold for growth by rectified diffusion (labeled RD, calculated for a variety of dissolved gas concentrations), and the threshold for inertial cavitation (labeled IC, defined conventionally as an expansion of the bubble to a size greater than twice its equilibrium radius) $)^{51,52}$ are all plotted on the same graph in order to facilitate identification of bubble behaviors.

Figure 1 plots all three thresholds for an acoustic pressure of 1.0 MPa at $1 \mathrm{MHz}$ for a gas-saturated medium. Once nucleated, the only bubbles which will survive are those 


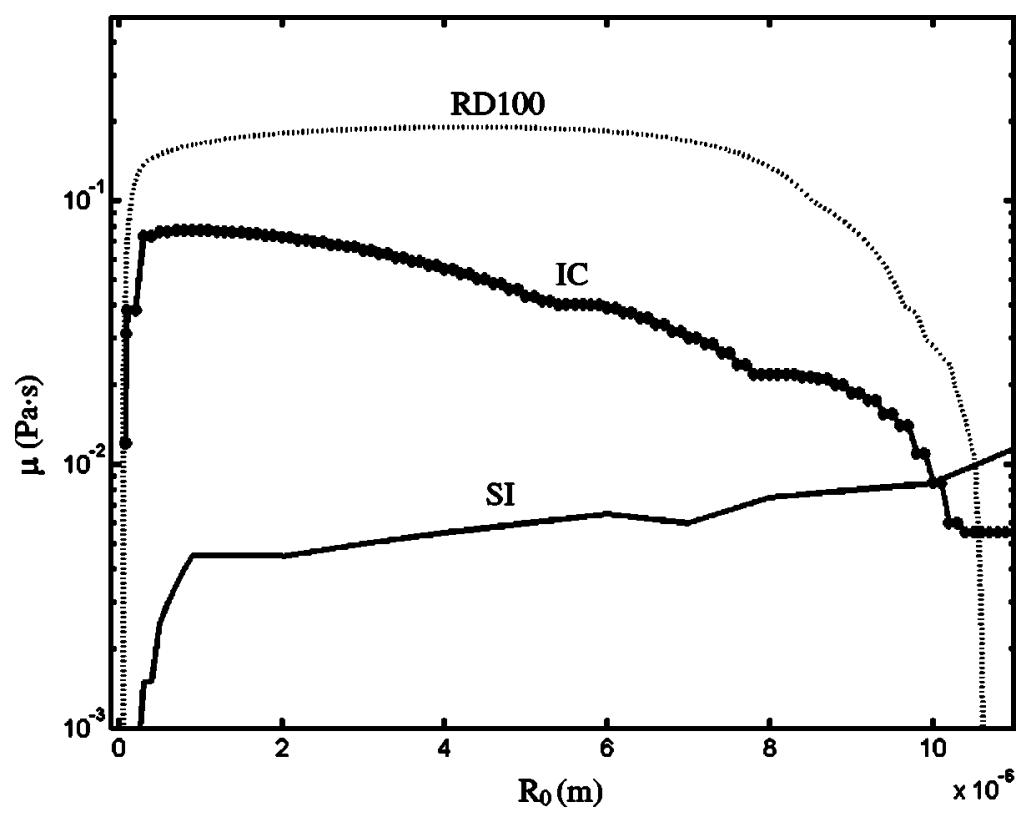

FIG. 1. $P_{a}=1.0 \mathrm{MPa}, f=1 \mathrm{MHz}$, and $100 \%$ gas saturation. Rectified diffusion (RD100, dashed line), shape instability (SI, thin solid line), and inertial cavitation (IC, thick solid line with stars) thresholds. Below RD bubbles grow. Below IC bubbles collapse inertially. Below SI bubbles break up from shape instabilities.

which are diffusively stable (on the RD threshold), or those which grow. In this space, a positive slope on the RD threshold line represents an unstable equilibrium, and a negative slope is stable. Bubbles below the RD threshold grow. Breakup via SIs bounds the region of surviving bubbles-all bubbles below the SI threshold line exceed the criterion for breakup via SI. Finally, since the experimental evidence of coincidence of broadband noise emissions detected by PCD always accompanies the observation of the onset of enhanced heating, we conclude that some fraction of the cavitation bubbles are collapsing inertially. The IC threshold line represents a minimal threshold for inertial collapse, and thus represents a boundary for the initial stages of cavitation activity. Thus, there is a wedge-shaped region in the parameter space for allowed bubble sizes, which in fact encompasses bubbles between 0.02 and $10 \mu \mathrm{m}$.

Figure 2 plots all three thresholds for an acoustic pressure of $2.5 \mathrm{MPa}$ at $1 \mathrm{MHz}$ for a gas-saturated medium. The situation is somewhat more complicated. The shape stable and shape unstable regions for this higher pressure are no longer simply connected; note the islands of instability for small bubbles between viscosity values 0.02 and $0.005 \mathrm{Pas}$. The size of the allowed region has increased, and admits bubbles with radii as small as $10 \mathrm{~nm}$ and as large as $17 \mu \mathrm{m}$.

Figure 3 shows the effect of changing dissolved gas concentration. When the gas concentration decreases from $100 \%$ to $10 \%$, the threshold does not shift very much, but from $10 \%$ to $0.1 \%$, there is a large difference between the calculated thresholds, with the growth region dramatically shrinking for decreasing dissolved gas concentration. One expects to find nearly saturated media in the body. However, for violent enough bubble collapse, sonoluminescence results ${ }^{42,40}$ have shown that only the noble gases participate in diffusion; $\mathrm{N}_{2}$ and $\mathrm{O}_{2}$ are dissociated during collapse and chemically bonded in water. This effect would expand the potential range of effective dissolved gas concentrations for

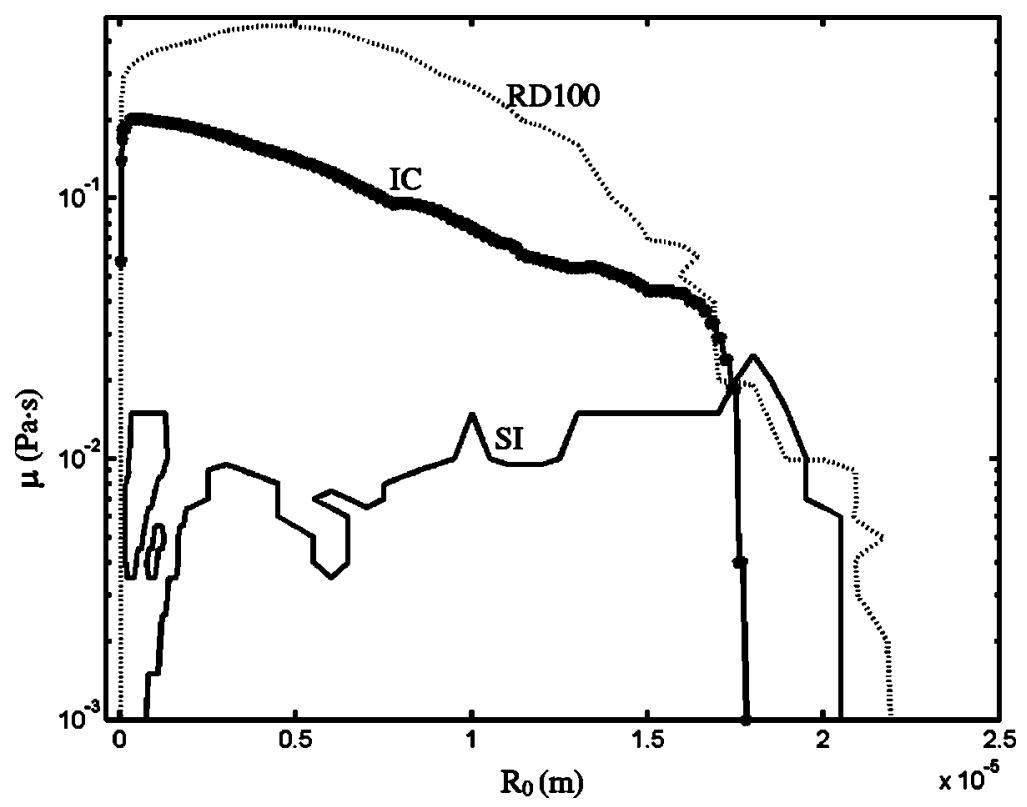

FIG. 2. $P_{a}=2.5 \mathrm{MPa}, f=1 \mathrm{MHz}$, and $100 \%$ gas saturation. Rectified diffusion (RD100, dashed line), shape instability (SI, thin solid line), and inertial cavitation (IC, thick solid line with stars) thresholds at $P_{a}$ $=1 \mathrm{MPa}, f=1 \mathrm{MHz}$. Below RD bubbles grow. Below IC bubbles collapse inertially. Below and enclosed by SI bubbles break up from shape instabilities. 


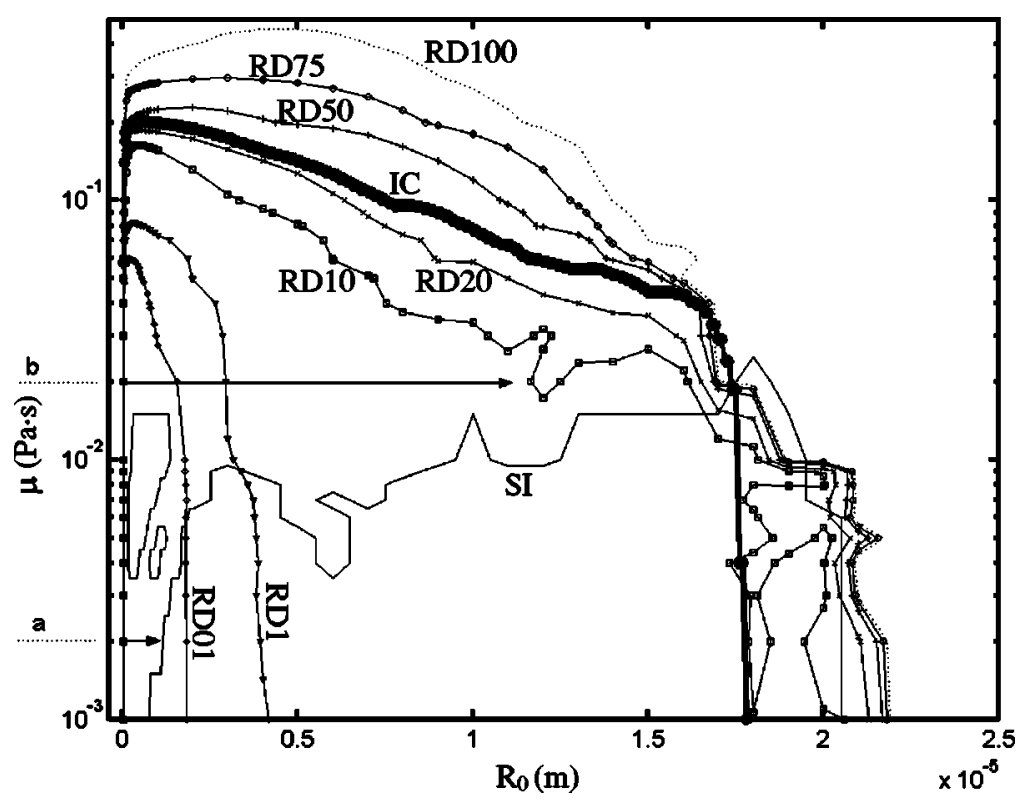

FIG. 3. $P_{a}=2.5 \mathrm{MPa}, f=1 \mathrm{MHz}$, and variable dissolved gas concentration. The inertial cavitation (IC) and shape instability (SI) thresholds are as in Fig. 2. All other lines are the RD thresholds for dissolved gas concentrations of $100 \%$ (RD100), $75 \%$ (RD75), $50 \%$ (RD50), 20\% (RD20), 10\% (RD10), 1\% (RD1), and $0.1 \%$ (RD01) relative to saturation. The two arrows show two paths for qualitatively different bubble behavior. For these two paths, the viscosities are (a) 0.002 Pa s; and (b) $0.02 \mathrm{~Pa}$ s. The total growth times for each path when the dissolved gas concentration is $10 \%$ are (a) $1.38 e-5 \mathrm{~s}$; and (b) $1.57 \mathrm{~s}$. The initial radius for both paths is $R_{0}(t=0)=50 \mathrm{~nm}$.

cavitation zone bubble dynamics to the well-undersaturated values calculated in Fig. 3. Such an effect, if it plays a role, would thus further limit the allowed bubble sizes to smaller bubbles. Choosing the $0.1 \%$ case for a most dramatic case illustration, while the lower limit of allowed bubble sizes remains virtually unchanged, the upper limit decreases to (for any viscosity value) less than $2.5 \mu \mathrm{m}$, meaning that in this case all allowed bubbles are below resonant size at 1 $\mathrm{MHz}$. Such a qualitative difference (from the saturated case) would profoundly affect the effective sound speed in the cavitation zone and thus would affect the primary HIFU propagation.

Figure 3 also shows the paths of two bubbles with initial radius of $50 \mathrm{~nm}$ in a $10 \%$ saturated medium which have two very different eventual fates. For the path labeled "a", we have chosen a viscosity of twice that of water. This bubble grows very quickly and in roughly 14 cycles becomes shape unstable. Such a bubble will break up, and likely one or more fragments will repeat this process of growth and breakup. For the path labeled " $b$ ", we have chosen a viscosity of 20 times that of water. This bubble grows more slowly, and eventually (after roughly $2 \mathrm{~s}$ ) encounters the stable branch of the rectified diffusion threshold, where it will remain since it is shape stable.

\section{B. Bubble size evolution}

Based on the wide variety of bubble dynamics behaviors (from inertial collapse to sinusoidal oscillations, from periodic to chaotic motion) encountered in this parameter space, one would expect and in fact observes in computation, a large change in growth rates for different bubble sizes. Figure 4 plots growth rates for growing bubbles at $3 \mathrm{MPa}$ and $100 \%$ saturation. Though the growth is rather modest for higher viscosity and larger bubbles sizes, the effect of inertial bubble dynamics (expansion of up to 80 times the initial

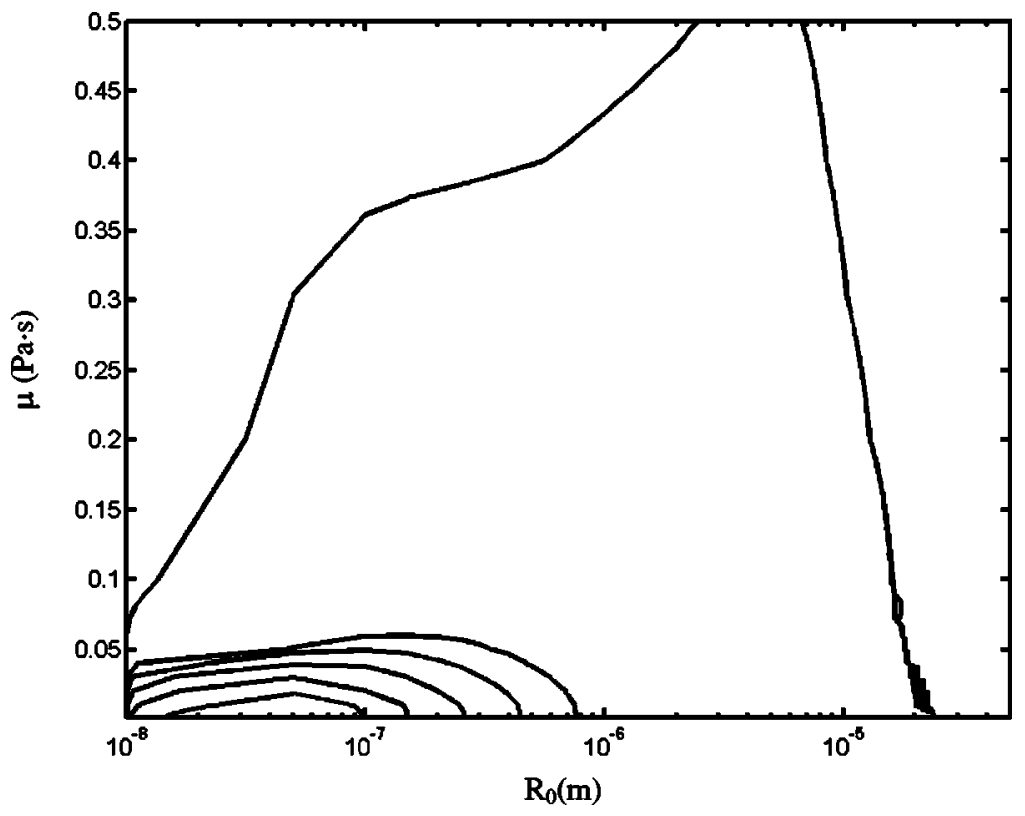

FIG. 4. Contour plot of bubble growth rates at $3 \mathrm{MPa}$, $1 \mathrm{MHz}$, and dissolved gas concentration $100 \%$. Solid lines correspond to growth rates of, from top to bottom, $0,1,10,100,1000$, and $10000 \mathrm{~m} / \mathrm{s}$. 

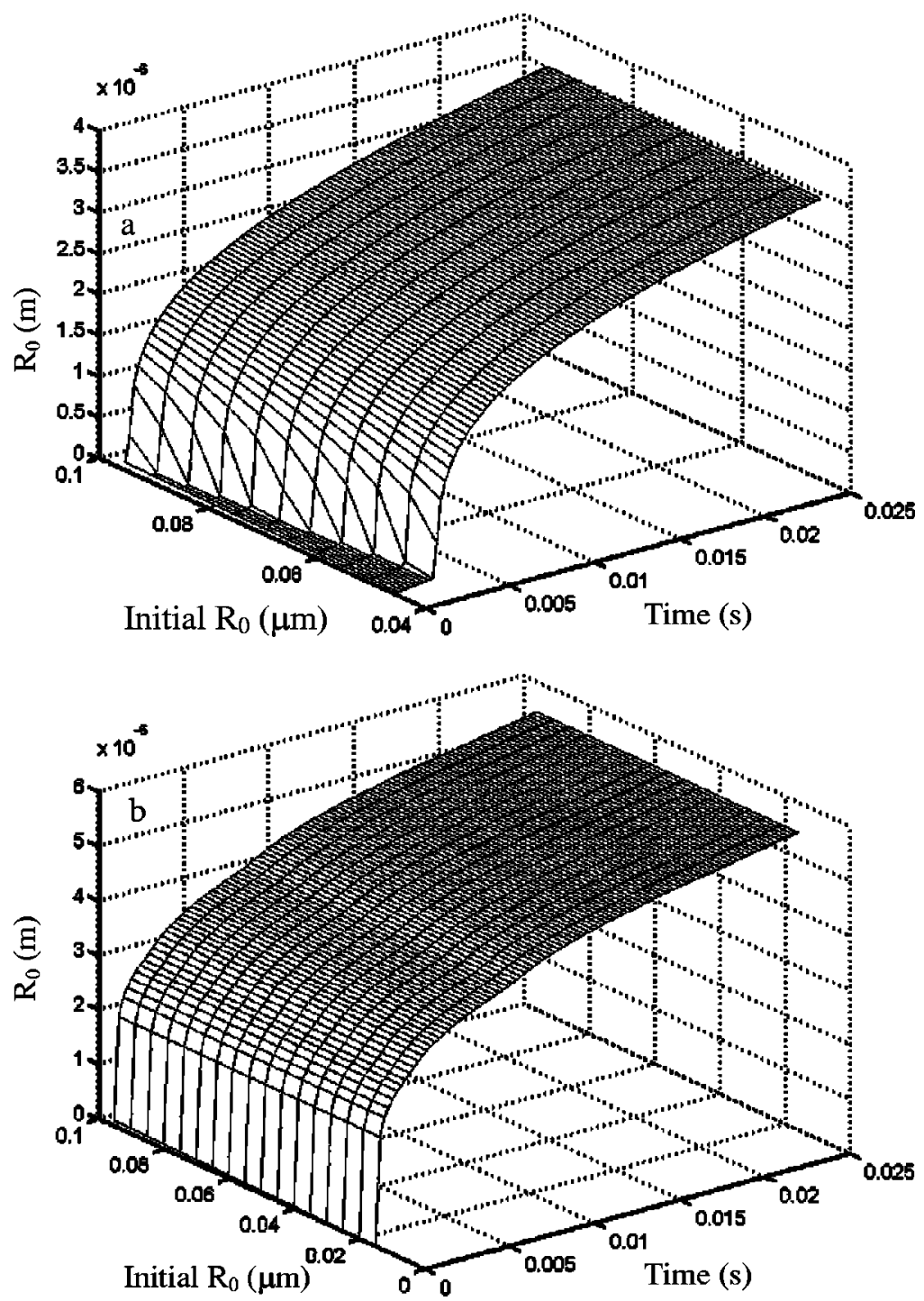

FIG. 5. Bubble equilibrium radius vs time for bubbles with initial radii ranging from $15 \mathrm{~nm}$ to $95 \mathrm{~nm}$ at $2 \mathrm{MPa}$ for different viscosities: (a) 0.05 Pa s and (b) $0.005 \mathrm{~Pa} \mathrm{~s}$. Dissolved gas concentration $20 \%$. radius followed by violent collapse in a single cycle) is clearly seen in the bunching of the growth rate contours for small (10 to $100 \mathrm{~nm}$ ) bubbles and small (0.001 to $0.02 \mathrm{~Pa} \mathrm{~s})$ viscosities.

The question arises as to whether or not this disparity of growth rates has implications for the temporal evolution of bubble size distributions. To investigate this question, we followed the growth of the bubble equilibrium radius for an array of initial bubble equilibrium sizes from $15 \mathrm{~nm}$ to 100 $\mathrm{nm}$ undergoing a $2 \mathrm{MPa}$ insonation in a $20 \%$ saturated medium. A "rectangular" distribution [full width at half maximum $(\mathrm{FWHM}) \sim \mathrm{O}(1)]$ corresponding to 1 bubble at each size in the range was used, and all initial $R_{0}$ are above the growth threshold. The results are shown in Fig. 5. In Fig. 5(a), when the viscosity is $0.05 \mathrm{~Pa} \mathrm{~s}$, the first few time slices reveal that, owing to the different growth rates experienced by the small and large initial bubbles, a bimodal distribution has developed. However, soon thereafter (by $5 \mathrm{~ms}$ ) the initially slow-growing bubbles have caught up, so that by the end of the $25 \mathrm{~ms}$ interval the profile is very nearly flat again. The mean radius is now, however, more than an order of magnitude greater at roughly $3.5 \mu \mathrm{m}$, so the FWHM at the end of the $25 \mathrm{~ms}$ interval is $\mathrm{O}\left(10^{-1}\right)$.
Figure 5(b) plots the same evolution when the viscosity is an order of magnitude lower (now five times that of water). The flat distribution evolves smoothly, and (though not seen on this plot) the differences between bubble equilibrium radii actually decrease from their initial value of $\Delta R_{0}$ $=5 \mathrm{~nm}$ during the $25 \mathrm{~ms}$ insonation, resulting in a FWHM at the end of $25 \mathrm{~ms}$ that is $\mathrm{O}\left(10^{-2}\right)$. Thus, for this range of viscosity values, an initial distribution of practically any character would narrow as the insonation progressed, so that any disperse distribution would be smoothed out by the growth dynamics into a nearly monodisperse distribution.

\section{DISCUSSION: WHICH BUBBLES WILL CONTRIBUTE?}

The main purpose of this study was to investigate bubble dynamics in a large parameter space relevant to HIFU, and if possible, bound the bubble size distribution that contributes to the enhanced heating observed in experiments. Only bubbles that do not dissolve and that are shape stable will contribute to the heating. Bubbles which contribute to heat generation will thus be trapped in the wedge-shaped region between the two instability thresholds, perhaps further con- 
strained by the IC threshold though our experimental knowledge is too incomplete to allow a stronger statement.

Two possible asymptotic behaviors naturally divide this allowed bubble region into two areas. In the lower area, characterized by viscosities on the order of water and illustrated by the path marked "a" in Fig. 3, bubbles will grow (rapidly) until they encounter the shape instability threshold and break up, whereupon a fragment may repeat the process. Such bubbles expand during one cycle to roughly 20 or more times their initial size and collapse inertially, breaking up typically via the Rayleigh-Taylor mechanism. To make any quantitative statement about the bubble sizes thus allowed one has to specify the acoustic pressure and frequency. For example, for $2.5 \mathrm{MPa}$ and $1 \mathrm{MHz} \mathrm{HIFU}$ in a material whose viscosity coefficient is between $0.001 \mathrm{~Pa} \mathrm{~s}$ (water) and 0.008 Pa s (whole blood at low shear rates), we may expect bubbles to be distributed (unevenly, perhaps) between $10 \mathrm{~nm}$ and roughly $1 \mu \mathrm{m}$.

For higher values of viscosity in the upper area, illustrated by the path marked "b", bubbles will grow until they become trapped on the stable (since it has a negative slope) rectified diffusion threshold, and these bubbles will stably oscillate at that size. In this regime, the dissolved gas concentration becomes the dominant factor in determining the upper size limit. Once again one has to specify parameters to infer a bubble size distribution. For $2.5 \mathrm{MPa}$ and $1 \mathrm{MHz}$ HIFU in a material whose apparent viscosity coefficient is between $0.02 \mathrm{Pas}$ (synovial fluid at moderate shear rates) and $0.2 \mathrm{Pas}$ (SAE 20 oil), we may expect bubbles to be distributed between a common lower bound and an upper bound determined by the dissolved gas concentration. The lower the dissolved gas concentration, the lower the upper bound of bubble sizes. To cite two extreme cases, for $0.1 \%$ saturation, the bubble size distribution will range from about $10 \mathrm{~nm}$ to only $2 \mu \mathrm{m}$. For $100 \%$ saturation, bubbles could grow as large as $18 \mu \mathrm{m}$, many times the (linear) resonant size.

The disconnected shape instability regions in Fig. 3 are caused by the strong nonlinearity of the oscillating bubbles. In all simulations, we use the same initial conditions: $R(0)$ $=R_{0}$ and $\dot{R}(0)=0$. For these strong nonlinear oscillating bubbles, different initial conditions may yield different radial motions; i.e., it is very likely that there are multiple coexisting steady-state solutions (or attractors) ${ }^{53,54}$ Such solutions are only elicited (at fixed parameter values) if different initial conditions are used. Thus, one would expect small shifts in the calculated shape stability threshold for calculations imposing different initial conditions. Such shifts will typically be small compared to any experimental uncertainties in parameters and measurements.

The calculated growth rates for small bubbles just above the Blake threshold are dramatically high, implying that such bubbles survive only a few cycles. However, the calculation of the growth rate has used the equilibrium value of bubble oscillation, averaged over a cycle. These facts suggest that a steady-state time-average calculation cannot be applied to these small bubbles without large errors, because the fast growth ensures large departures from equilibrium. Though beyond the scope of this work, a more accurate treatment of these bubbles will be found by relaxing the implicit assumption that the smooth part of the mass diffusion problem is dominant (see Refs. 48 and 50 for details). For the larger viscosity regions of parameter space, the contribution from these small bubbles will be small since bubbles will grow and pass that region in a matter of a few cycles. For the small viscosity regions, whether a bubble survives one cycle or ten will also be unimportant as long as the problems of interest require hundreds or more cycles of oscillation. Thus, these calculations should be valid for therapeutic situations providing the ultrasound burst is at least 100 cycles or $100 \mu$ s at 1 $\mathrm{MHz}$.

We used a Newtonian model to investigate the bubble dynamics. We know however that real tissue materials are viscoelastic. We investigated a large range of viscosity to try to cover many of the possibilities. However, how good (or bad) this approach is needs to be determined, and this requires a numerically tractable non-Newtonian bubble model ${ }^{55,56}$ Another practical difficulty related to this problem is that the viscoelastic material properties of tissue, in general, are difficult to obtain, and moreover are strain-type and strain-rate dependent. Bubbles present a dilatational strain, and nonlinearly varying strain rates (over a single cycle of motion in many cases). Ultimately, there is a need for an explicit experimental investigation of bubble dynamics in viscoelastic media, including tissue.

\section{CONCLUSION}

These bubble dynamics results over a broad parameter range allow an experimenter to narrow down the possibilities for bubble sizes as long as some information about the parameters $\left(P_{a}, f, \mu\right.$, and $\left.c_{i} / c_{0}\right)$ of a given experiment is available. The results may then be applied to calculate the bubbles contribution to the heat deposition during therapeutic ultrasound treatment. For example, in the series of tissuemimicking experiments, ${ }^{10,11,57}$ the investigators found that, by applying these results to the interpretation of their experiments, they could infer that only something on the order of 50 appropriately sized bubbles were required to account for the observed peak enhanced heating. Moreover, since the models as presented here are complete (they capture the dominant physical phenomena of nontranslating bubbles in a sound field) and numerically practical, they may be adopted as tools for investigation of bubble effects (including heating) for other situations requiring predictive characterization of ultrasound applications.

\section{ACKNOWLEDGMENTS}

The authors gratefully acknowledge the support of NASA, DARPA (via a subcontract to the University of Washington Applied Physics Laboratories), and the U.S. Army (via a subcontract to the University of Mississippi National Center for Physical Acoustics). The suggestions and support of the PACLAB group at BU are also gratefully acknowledged. This research was supported through award No. DAMD17-02-2-0014, administered by the U.S. Army Medical Research Acquisition Activity, Fort Detrick, MD. The 
content of this paper does not necessarily reflect the position of the policy of the Government, and no official endorsement should be inferred.

${ }^{1}$ E. N. Harvey, "Biological aspects of ultrasonic waves, a general survey," Biol. Bull. 59, 306-325 (1930).

${ }^{2}$ T. F. Hueter, H. T. Ballantine, and W. C. Cotter, "Production of lesions in the central nervous system with focused ultrasound: A study of dosage factors," J. Acoust. Soc. Am. 28, 192-201 (1956).

${ }^{3}$ F. J. Fry, R. F. Heimburger, L. V. Gibbons, and R. C. Eggleton, "Ultrasound for visualization and modification of brain tissue," IEEE Trans. Sonics Ultrason. SU-17, 165-169 (1970).

${ }^{4}$ P. P. Lele, "Effects of ultrasound on solid mammalian tissues and tumors in vivo," Ultrasound: Medical Applications, Biological Effects and Hazard Potential (Plenum, New York, 1987), pp. 275-306.

${ }^{5} \mathrm{~K}$. Hynynen, "The threshold for thermally significant cavitation in dog's thigh muscle in vivo," Ultrasound Med. Biol. 17, 157-169 (1991).

${ }^{6}$ D. L. Miller and R. A. Gies, "The interaction of ultrasonic heating and cavitation in vascular bioeffects on mouse intestine," Ultrasound Med. Biol. 24, 123-128 (1998).

${ }^{7}$ S. Vaezy, X. Shi, R. W. Martin, E. Chi, P. I. Nelson, M. R. Bailey, and L. A. Crum, "Real-time visualization of focused ultrasound therapy," Ultrasound Med. Biol. 27, 33-42 (2001)

${ }^{8}$ N. I. Vykhodtseva, K. Hynynen, and C. Damianou, "Histologic effects of high intensity pulsed ultrasound exposure with subharmonic emission in rabbit brain in vivo," Ultrasound Med. Biol. 21, 969-979 (1995).

${ }^{9}$ G. R. ter Haar and S. Daniels, "Evidence for ultrasonically induced cavitation in vivo," Phys. Med. Biol. 26, 1145-1149 (1981).

${ }^{10}$ R. G. Holt and R. A. Roy, "Measurements of bubble-enhanced heating from focused, MHz-frequency ultrasound in a tissue-mimicking material," Ultrasound Med. Biol. 27, 1399-1412 (2001).

${ }^{11} \mathrm{P}$. L. Edson, "The role of acoustic cavitation in enhanced ultrasoundinduced heating in a tissue-mimicking phantom," Ph.D. dissertation, Boston University, 2001.

${ }^{12}$ R. A. Roy, S. I. Madanshetty, and R. E. Apfel, "An acoustic backscattering technique for the detection of transient cavitation produced by microsecond pulses of ultrasound," J. Acoust. Soc. Am. 87, 2451-2458 (1990).

${ }^{13}$ M. R. Bailey, L. N. Couret, O. A. Spaozhnikov, V. A. Khokhlova, G. R. ter Haar, S. Vaezy, X. Shi, R. Martin, and L. A. Crum, "Use of overpressure to assess the role of bubbles in focused ultrasound lesion shape in vitro," Ultrasound Med. Biol. 27, 695-708 (2001).

${ }^{14}$ S. D. Sokka, R. King, and K. Hynynen, "MRI-guided gas bubble enhanced ultrasound heating in in vivo rabbit thigh," Phys. Med. Biol. 48, 223-241 (2003)

${ }^{15}$ S. Hilgenfeldt, D. Lohse, and M. Zomack, "Sound scattering and localized heat deposition of pulse-driven microbubbles," J. Acoust. Soc. Am. 107, 3530-3539 (2000)

${ }^{16} \mathrm{~S}$. Hilgenfeldt, D. Lohse, and M. Zomack, "Response of bubbles to diagnostic ultrasound: A unifying theoretical approach," Eur. Phys. J. B 4, 247-255 (1998)

${ }^{17}$ F. Chavrier, J. Y. Chapelon, A. Gelet, and D. Cathignol, "Modeling of high-intensity focused ultrasound-induced lesions in the presence of cavitation bubbles," J. Acoust. Soc. Am. 108, 432-440 (2000).

${ }^{18}$ A. A. Brayman, M. Azadniv, C. Cox, and M. W. Miller, "Hemolysis of albunex-supplemented, $40 \%$ hematocrit human erythrocytes in vitro by 1 MHz pulsed ultrasound: Acoustic pressure and pulse length dependence," Ultrasound Med. Biol. 22, 927-938 (1996).

${ }^{19}$ S. L. Poliachik, W. L. Chandler, P. D. Mourad, M. R. Bailey, S. Bloch, R. O. Cleveland, P. Kaczkowski, G. Keilman, T. Porter, and L. A. Crum, "Effect of high-intensity focused ultrasound on whole blood with and without microbubble contrast agent," Ultrasound Med. Biol. 25, 991-998 (1999).

${ }^{20}$ D. L. Miller and R. A. Gies, "Enhancement of ultrasonically-induced hemolysis by perfluorocarbon-based compared to air-based echo-contrast agents," Ultrasound Med. Biol. 24, 285-292 (1998).

${ }^{21}$ M. W. Miller, E. C. Everbach, C. Cox, R. R. Knapp, A. A. Brayman, and T. A. Sherman, "A comparison of the hemolytic potential of optison and albunex in whole human blood in vitro: Acoustic pressure, ultrasound frequency, donor and passive cavitation detection considerations," Ultrasound Med. Biol. 27, 709-721 (2001).

${ }^{22}$ S. Vaezy, R. Martin, U. Schmiedl, M. Caps, S. Taylor, K. Beach, S. Carter, P. Kaczkowski, G. Keilman, S. Helton, W. Chandler, P. Mourad, M. Rice, R. A. Roy, and L. A. Crum, "Liver hemostasis using high-intensity fo- cused ultrasound," Ultrasound Med. Biol. 23, 1413-1420 (1997).

${ }^{23}$ S. L. Poliachik, W. L. Chandler, P. D. Mourad, R. J. Ollos, and L. A. Crum, "Activation, aggregation and adhesion of platelets exposed to highintensity focused ultrasound," Ultrasound Med. Biol. 27, 1567-1576 (2001).

${ }^{24}$ M. L. Noble, S. Vaezy, A. Keshavarzi, M. Paun, A. F. Prokop, E. Y. Chi, C. Cornejo, S. R. Sharar, G. J. Jurkovich, R. W. Martin, and L. A. Crum, "Spleen hemostasis using high-intensity ultrasound: Survival and healing," J. Trauma: Inj., Infect., Crit. Care 53, 1115-1120 (2002).

${ }^{25} \mathrm{~K}$. Y. Ng and Y. Liu, "Therapeutic ultrasound: Its application in drug delivery," Med. Res. Rev. 22, 204-223 (2002).

${ }^{26}$ C. Lauer, R. Burge, D. B. Tang, B. G. Bass, E. R. Gomez, and B. B. Alving, "Effect of ultrasound on tissue-type plasminogen activatorinduced thrombolysis," Circulation 86, 1257-1264 (1992).

${ }^{27}$ C. W. Francis, P. T. Onundarson, E. L. Carstensen, A. Blinc, R. S. Meltzer, K. Schwarz, and V. J. Marder, "Enhancement of fibrinolysis in vitro by ultrasound," J. Clin. Invest. 90, 2063-2068 (1992).

${ }^{28}$ W. C. Culp, T. R. Porter, F. Xie, T. C. Goertzen, T. C. McCowan, B. N. Vonk, and B. T. Baxter, "Microbubble potentiated ultrasound as a method of declotting thrombosed dialysis grafts: Experimental study in dogs," Cardiovasc. Intervent Radiol. 24, 407-412 (2001)

${ }^{29}$ B. Devcic-Kuhar, S. Pfaffenberger, M. Grö Schl, C. Kollmann, E. Benes, and M. Gottsauner-Wolf, "In vitro thrombolysis enhanced by standing and traveling ultrasound wave fields," Ultrasound Med. Biol. 28, 1181-1187 (2002)

${ }^{30}$ Y. Adler, A. Dagan, G. Golovchiner, Z. Iakobishvili, I. Matz, E. Lev et al., "Augmentation of low-frequency ultrasound-induced clot disruption by hydroxyethyl starch is dependent on the duration and intensity of ultrasound exposure; An in vitro study," Ultrasound Med. Biol. 29, 483-486 (2003)

${ }^{31}$ A. V. Alexandrov, A. M. Demchuk, M. D. Hill, R. A. Felberg, O. Chernyshev, Z. Garami et al., "CLOTBUST: Phase I data on ultrasound enhanced thrombolysis for stroke," Stroke 33, 72 (2002).

${ }^{32}$ T. G. Leighton, The Acoustic Bubble (Academic, New York, 1994).

${ }^{33}$ J. B. Keller and M. J. Miksis, "Bubble oscillations of large amplitude," J. Acoust. Soc. Am. 68, 628-633 (1980).

${ }^{34}$ A. Prosperetti and A. Lezzi, "Bubble dynamics in a compressible liquid. I: First-order theory," J. Fluid Mech. 168, 457-478 (1986).

${ }^{35}$ A. Prosperetti, L. A. Crum, and K. W. Commander, "Nonlinear bubble dynamics," J. Acoust. Soc. Am. 83, 502-514 (1988).

${ }^{36} \mathrm{~V}$. Kamath and A. Prosperetti, "Numerical integration methods in gasbubble dynamics," J. Acoust. Soc. Am. 85, 1538-1548 (1989).

${ }^{37}$ M. S. Plesset, "On the stability of fluid flows with spherical symmetry," J. Appl. Phys. 25, 96-98 (1954).

${ }^{38}$ A. Eller and L. A. Crum, "Instability of the motion of a pulsating bubble in a sound field," J. Acoust. Soc. Am. 47, 762-767 (1970).

${ }^{39}$ A. Prosperetti, "On the stability of spherically symmetric flows," Atti Accad. Naz. Lincei, Rend. Cl. Sci. Fis. Mat. Nat. 62, 196-203 (1977).

${ }^{40}$ S. Hilgenfeldt, D. Lohse, and M. P. Brenner, "Phase diagrams for sonoluminescing bubbles," Phys. Fluids 8, 2808-2826 (1996).

${ }^{41}$ Y. Hao and A. Prosperetti, "The effect of viscosity on the spherical stability of oscillating gas bubbles," Phys. Fluids 11, 1309-1317 (1999).

${ }^{42}$ R. G. Holt and D. F. Gaitan, "Observation of stability boundaries in the parameter space of single bubble sonoluminescence," Phys. Rev. Lett. 77, 3791-3794 (1996).

${ }^{43}$ D. F. Gaitan and R. G. Holt, "Experimental observations of bubble response and light intensity near the threshold for single bubble sonoluminescence in an air-water system," Phys. Rev. E 59, 5495-5502 (1999).

${ }^{44}$ A. Prosperetti and Y. Hao, "Modeling of spherical gas bubble oscillations and sonoluminescence," Philos. Trans. R. Soc. London, Ser. A 357, $203-$ 223 (1999)

${ }^{45}$ A. Prosperetti, "On the stability of spherically symmetric flows," Atti Accad. Naz. Lincei, Rend. Cl. Sci. Fis. Mat. Nat. 62, 196-203 (1977).

${ }^{46}$ M. P. Brenner, D. Lohse, and T. F. Dupont, "Bubble shape oscillations and the onset of sonoluminescence," Phys. Rev. Lett. 75, 954-957 (1995).

${ }^{47}$ A. Eller and H. G. Flynn, "Rectified diffusion during nonlinear pulsations of cavitation bubbles," J. Acoust. Soc. Am. 37, 493-503 (1965).

${ }^{48}$ M. M. Fyrillas and A. J. Szeri, "Dissolution or growth of soluble spherical oscillating bubbles," J. Fluid Mech. 277, 381-407 (1994).

${ }^{49}$ J. Huang, "Heating in vascular and flow-through tissue phantoms induced by focused ultrasound," Ph.D. dissertation, Boston University, 2002.

${ }^{50} \mathrm{X}$. Yang, "Investigation of bubble dynamics and heating during focused ultrasound insonation in tissue-mimicking materials," Ph.D. dissertation, Boston University, 2003. 
${ }^{51}$ H. G. Flynn, "Cavitation dynamics. II: Free pulsations and models for cavitation bubbles," J. Acoust. Soc. Am. 58, 1160-1170 (1975).

${ }^{52}$ H. G. Flynn and C. C. Church, "Transient pulsations of small gas bubbles in water," J. Acoust. Soc. Am. 84, 985-998 (1988).

${ }^{53}$ U. Parlitz, V. Englisch, C. Scheffczyk, and W. Lauterborn, "Bifurcation structure of bubble oscillations," J. Acoust. Soc. Am. 88, 1061-1077 (1990).

${ }^{54}$ W. Lauterborn and J. Holzfuss, "Acoustic chaos," Int. J. Bifurcation Chaos Appl. Sci. Eng. 1, 13-26 (1991).
${ }^{55}$ J. S. Allen and R. A. Roy, "Dynamics of gas bubbles in viscoelastic fluids. I: Linear viscoelasticity," J. Acoust. Soc. Am. 107, 3167-3178 (2000).

${ }^{56}$ J. S. Allen and R. A. Roy, "Dynamics of gas bubbles in viscoelastic fluids. II: Nonlinear viscoelasticity," J. Acoust. Soc. Am. 108, 1640-1650 (2000)

${ }^{57}$ R. G. Holt, R. A. Roy, P. A. Edson, and X. Yang, "Bubbles and HIFU: The good, the bad, and the ugly," Proceedings of the Second International Symposium on Therapeutic Ultrasound, edited by L. Crum (2002), p. 120. 